\title{
Suppression of subcutaneous oxidation during the deposition of amorphous lanthanum aluminate on silicon
}

\author{
L. F. Edge and D. G. Schlom ${ }^{\text {a) }}$ \\ Department of Materials Science and Engineering, Pennsylvania State University, University Park, \\ Pennsylvania 16802 \\ R. T. Brewer and Y. J. Chabal \\ Departments of Chemistry and Chemical Biology, Biomedical Engineering and Physics, Rutgers University, \\ Piscataway, New Jersey 08854 \\ J. R. Williams and S. A. Chambers \\ Fundamental Science Directorate, Pacific Northwest National Laboratory, Richland, Washington 99352 \\ C. Hinkle and G. Lucovsky \\ Department of Physics, North Carolina State University, Raleigh, North Carolina 27695 \\ Y. Yang and S. Stemmer \\ Materials Department, University of California, Santa Barbara, California 93106 \\ M. Copel \\ IBM T. J. Watson Research Center, Yorktown Heights, New York 10598 \\ B. Holländer and J. Schubert \\ Institut für Schichten und Grenzfächen ISG1-IT and Center of Nanoelectronic Systems for Information \\ Technology, Forschungszentrum Jülich GmbH, D-52425 Jülich, Germany
}

(Received 10 December 2003; accepted 7 April 2004; published online 19 May 2004)

\begin{abstract}
Amorphous $\mathrm{LaAlO}_{3}$ thin films have been deposited by molecular beam deposition directly on silicon without detectable oxidation of the underlying substrate. We have studied these abrupt interfaces by Auger electron spectroscopy, high-resolution transmission electron microscopy, medium-energy ion scattering, transmission infrared absorption spectroscopy, and x-ray photoelectron spectroscopy. Together these techniques indicate that the films are fully oxidized and have less than $0.2 \AA$ of $\mathrm{SiO}_{2}$ at the interface between the amorphous $\mathrm{LaAlO}_{3}$ and silicon. These heterostructures are being investigated for alternative gate dielectric applications and provide an opportunity to control the interface between the silicon and the gate dielectric. (C) 2004 American Institute of Physics. [DOI: 10.1063/1.1759065]
\end{abstract}

The continued scaling of $\mathrm{SiO}_{2}$ in metal-oxidesemiconductor field-effect transistors (MOSFETs) is approaching the fundamental thickness limit of $7 \AA .{ }^{1}$ Below this thickness, $\mathrm{SiO}_{2}$ is predicted to no longer be an effective gate insulator. ${ }^{1}$ One way to circumvent this problem is to replace $\mathrm{SiO}_{2}$ with a gate dielectric having a higher dielectric constant such as $\mathrm{LaAlO}_{3} \cdot{ }^{2-4}$ Amorphous $\mathrm{LaAlO}_{3}$ thin films on silicon have an estimated dielectric constant of $20-27^{5-7}$ and an optical band gap of $6.2 \pm 0.1{ }^{8}$ The band offsets between amorphous $\mathrm{LaAlO}_{3}$ and silicon were recently measured to be $1.8 \pm 0.2 \mathrm{eV}$ and $3.2 \pm 0.1 \mathrm{eV}$ for the conduction and valence bands, respectively. ${ }^{8}$ It has also been shown that single crystalline $\mathrm{LaAlO}_{3}$ is stable in contact with silicon under standard MOSFET processing conditions of $1026^{\circ} \mathrm{C}$ for 20 s. $^{9}$ These known properties make $\mathrm{LaAlO}_{3}$ a promising candidate material to replace $\mathrm{SiO}_{2}$ in MOSFETs.

One of the major challenges ${ }^{10}$ in producing an alternative gate dielectric is the ability to grow very thin alternative gate dielectrics on silicon without forming unwanted $\mathrm{SiO}_{2}$ at the interface during deposition and without the need to cap the film in situ to prevent such $\mathrm{SiO}_{2}$ formation upon air exposure after growth. ${ }^{11-16}$ Only a few groups have reported the growth of gate dielectrics on silicon without substantial

${ }^{a)}$ Electronic mail: schlom@ems.psu.edu formation of interfacial $\mathrm{SiO}_{2}$ without capping the film before exposure to air. ${ }^{17-24}$ The thinnest of these films was $22 \AA$ of amorphous $\mathrm{Al}_{2} \mathrm{O}_{3}$ on silicon. ${ }^{18}$

In this letter, we present the growth of amorphous thin films of $\mathrm{LaAlO}_{3}$ directly on (001) Si by molecular beam deposition without the formation of $\mathrm{SiO}_{2}$ at the interface. The films were deposited by codeposition of lanthanum, aluminum, and oxygen at the minimum partial pressure of oxygen required to fully oxidized the metal species. This minimum oxygen pressure was established by detailed oxidation experiments of the constituents of $\mathrm{LaAlO}_{3}$, which are reported elsewhere. ${ }^{25}$ Even after prolonged air exposure of films as thin ${ }^{26}$ as $10 \AA$ there was no formation of $\mathrm{SiO}_{2}$ or $\mathrm{SiO}_{x}$ at the interface within the maximum sensitivity of our methods $(\sim 0.2 \AA)$.

Amorphous $\mathrm{LaAlO}_{3}$ films of thickness ${ }^{26}$ equal to 10 and $40 \AA$ were grown by molecular beam deposition (MBD) in an EPI 930 MBE chamber modified for the growth of oxides. ${ }^{27}$ The films for transmission infrared absorption spectroscopy were grown on one side of double-side polished $p$-type (001) Si (boron doped, $<1.4 \times 10^{15} \mathrm{~B} / \mathrm{cm}^{-3}$ ). The films for the other measurements were grown on $p$-type (001) $\mathrm{Si}$ [boron doped $(3-6) \times 10^{15} \mathrm{~B} / \mathrm{cm}^{-3}$ ] with one side polished. The native $\mathrm{SiO}_{2}$ on the silicon wafer was thermally desorbed in ultrahigh vacuum at a substrate temperature of 


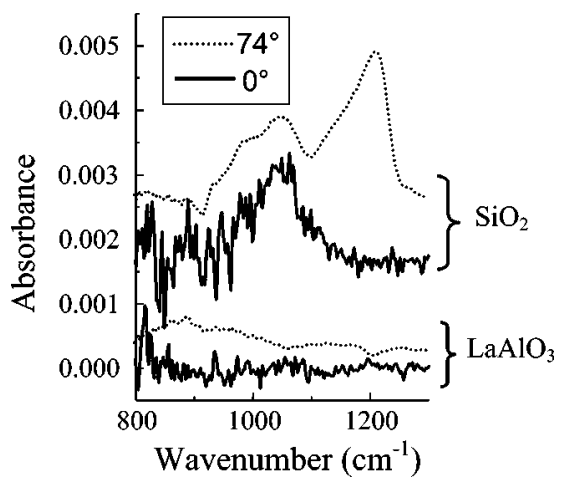

FIG. 1. Transmission infrared absorption spectra of $\sim 4 \AA \mathrm{SiO}_{2} / \mathrm{Si}$, using $\mathrm{H} / \mathrm{Si}$ as reference, and $10 \AA$ amorphous $\mathrm{LaAlO}_{3} / \mathrm{Si}$, using the sample after removal of the $\mathrm{LaAlO}_{3}$ film and $\mathrm{H}$-terminating the surface for reference (keeping the back surface constant). Data are taken at normal incidence and $74^{\circ}$ in both cases.

$900^{\circ} \mathrm{C}$, as measured with a pyrometer. The films were grown using elemental sources. Lanthanum, aluminum, and molecular oxygen ( $99.994 \%$ purity) at a background pressure of $6 \times 10^{-8}$ Torr, were codeposited with the substrate at a thermocouple temperature of $\sim 100^{\circ} \mathrm{C}$. The lanthanum and aluminum fluxes were each $2 \times 10^{13}$ atoms $/ \mathrm{cm}^{2} \mathrm{~s}$. Several $\sim 1000$ - $\AA$-thick amorphous $\mathrm{LaAlO}_{3}$ films were analyzed by Rutherford backscattering spectrometry (RBS) (1.4 MeV $\mathrm{He}+, 170^{\circ}$ scattering angle). These measurements revealed that the films were stoichiometric with a $1: 1 \mathrm{La}: \mathrm{Al}$ ratio to within $\pm 0.05 \mathrm{~mol} \%$.

The samples were grown at Penn State and then shipped in air to the other laboratories for additional measurements. Transmission infrared absorption spectroscopy was performed at Rutgers with a Nicolet Magna interferometer operated at $4 \mathrm{~cm}^{-1}$ resolution. ${ }^{28}$ The $\mathrm{x}$-ray photoelectron spectroscopy (XPS) core level spectra were obtained at PNNL with a GammaData SES 200 analyzer using monochromatic $\mathrm{Al} K \alpha \mathrm{x}$ rays at a normal emission geometry. The Auger electron spectroscopy (AES) spectra were obtained at NCSU using a Phi model 11-010 Auger electron spectrometer operated with a beam diameter and current that would minimize sample damage. High-resolution transmission electron microscopy (HRTEM) images were acquired at UCSB using a $200 \mathrm{kV}$ TEM (JEOL 2010). The medium-energy ion scattering (MEIS) analysis was completed at IBM with $100 \mathrm{keV}$ protons channeling at normal incidence and the backscattered protons were detected with a toroidal electrostatic analyzer at a scattering angle of $110^{\circ}$.

Figure 1 compares the transmission infrared absorption spectra (taken at both $74^{\circ}$ and $0^{\circ}$ incidence angles) of a 10$\AA$-thick $\mathrm{LaAlO}_{3}$ film deposited on silicon with the absorption spectra of a 4 - $\AA$-thick $\mathrm{SiO}_{2}$ layer on silicon [made by wet chemical treatment of hydrogen-terminated (001) $\mathrm{Si}$ in 4:1 $\mathrm{H}_{2} \mathrm{SO}_{4}: \mathrm{H}_{2} \mathrm{O}_{2}$ at $80^{\circ} \mathrm{C}$ for $\left.10 \mathrm{~min}\right]$. The transverse optical mode at $\sim 1050 \mathrm{~cm}^{-1}$ and the longitudinal optical mode at $\sim 1210 \mathrm{~cm}^{-1}$ are the signatures of the $4-\AA$-thick $\mathrm{SiO}_{2}$ film. Clearly, there is no evidence of these modes in the absorption spectra of the $\mathrm{LaAlO}_{3}$ film. However, difficulties related to the ill-defined back surface of the $\mathrm{LaAlO}_{3}$ wafer limit the signal-to-noise ratio, placing an upper limit of $0.2 \AA$ for a possible interfacial $\mathrm{SiO}_{2}$ layer. To alleviate this problem, transmission infrared absorption spectra were also taken

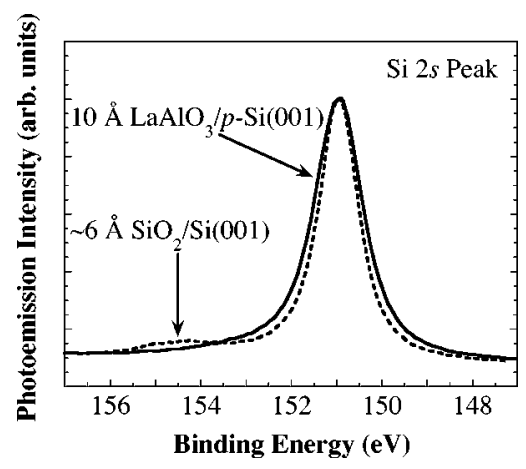

FIG. 2. High-energy resolution XPS core-level spectra of the $\mathrm{Si} 2 s$ region for a 10- $\AA$-thick amorphous $\mathrm{LaAlO}_{3}$ film on (001) $\mathrm{Si}$ in comparison with $\sim 6 \AA$ of $\mathrm{SiO}_{2}$ on (001) Si.

from 10 - $\AA$-thick amorphous $\mathrm{LaAlO}_{3} / \mathrm{Si}$ grown in an identical way, except that the (001) Si wafer was wet cleaned and hydrogen-terminated with HF before being loaded into the MBD growth chamber. This precleaning dramatically improved the quality of the back side of the wafer, making it possible to get a much better baseline and signal-to-noise ratio for the infrared measurements. With this sample, we also see no evidence for interfacial $\mathrm{SiO}_{2}$, and can now set an upper limit for interfacial $\mathrm{SiO}_{2}$ of $0.07 \AA$.

Figure 2 shows the Si $2 s$ peak measured by XPS from a 10- $\AA$-thick film of amorphous $\mathrm{LaAlO}_{3} / \mathrm{Si}$ along with that of $\sim 6 \AA$ of $\mathrm{SiO}_{2} / \mathrm{Si}$. There is clearly no peak at $\sim 155 \mathrm{eV}$ characteristic of $\mathrm{SiO}_{2}$ in the $\mathrm{LaAlO}_{3}$ spectrum, showing there is no detectable $\mathrm{SiO}_{2}$ at the interface. We estimate the detection limit to be $\sim 3 \AA$ of $\mathrm{SiO}_{2}$. The $\mathrm{Si} 2 p$ was not analyzed because the La $4 d$ peaks from the amorphous $\mathrm{LaAlO}_{3}$ thin film overlap the position where photoemission from $\mathrm{SiO}_{2}$ would be observed.

The same sample was then analyzed by AES. Figure 3 shows the AES spectrum, which shows no detectable $\mathrm{SiO}_{2}$ at the interface between amorphous $\mathrm{LaAlO}_{3}$ and silicon. There is a trace amount of chlorine present in the film. The chlorine is probably from the lanthanum source. Comparing this AES trace with studies of $\mathrm{SiO}_{2}$ formation by remote plasmaassisted oxidation, we estimate the sensitivity of AES to be $<3 \AA$ of $\mathrm{SiO}_{2}$ under a $10-\AA$-thick amorphous $\mathrm{LaAlO}_{3}$ film.

A cross-sectional TEM specimen was made from the same $10 \AA$ sample analyzed by XPS and AES. Figure 4 shows the HRTEM cross sectional image. No $\mathrm{SiO}_{2}$ or $\mathrm{SiO}_{x}$ is evident at the interface between the $\mathrm{LaAlO}_{3}$ film and the silicon and there are no contrast changes across the sample,

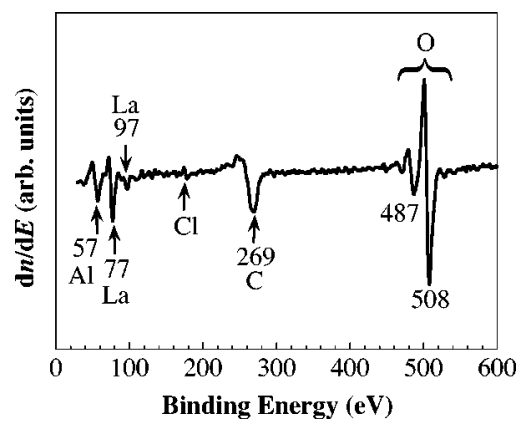

FIG. 3. AES spectra of a 10 - $\AA$-thick amorphous $\mathrm{LaAlO}_{3}$ film on (001) $\mathrm{Si}$

showing no detectable $\mathrm{SiO}_{2}$ 


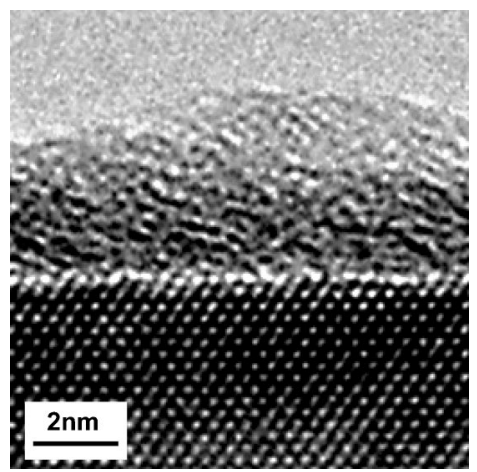

FIG. 4. Cross-sectional HRTEM image of $10 \AA$ of amorphous $\mathrm{LaAlO}_{3}$ on (001) Si showing no interfacial layer between the $\mathrm{LaAlO}_{3}$ film and silicon.

which indicates that there are no gross compositional variations across the sample.

The MEIS spectrum of a 40- $\AA$-thick $\mathrm{LaAlO}_{3}$ film on silicon, deposited in the same way as the other amorphous $\mathrm{LaAlO}_{3} / \mathrm{Si}$ samples, shows well separated lanthanum, aluminum/silicon, and oxygen backscatter peaks (Fig. 5). When the lanthanum and oxygen peaks are plotted on the same depth scale after corrections for cross section and concentration (see the inset), we see that the two elements have nearly identical depth distributions, indicating the near total absence of interfacial $\mathrm{SiO}_{2}$. Quantitative modeling confirmed this conclusion, to an upper limit of $2 \AA$ of interfacial $\mathrm{SiO}_{2}$. We note that the masses of aluminum and silicon are similar, making it difficult to discriminate between the two species in a buried layer using MEIS. Thus, although we can put tight constraints on the quantity of interfacial $\mathrm{SiO}_{2}$, there is a reduced sensitivity to interfacial $\mathrm{LaSiO}_{x}$ at the interface between the amorphous $\mathrm{LaAlO}_{3}$ and $\mathrm{Si}$. The ability to avoid subcutaneous oxidation provides an opportunity to controllably introduce $\mathrm{SiO}_{2}$ at the interface and study its effect on the electrical properties of the dielectric stack as a function of the interfacial $\mathrm{SiO}_{2}$ thickness.

L.F.E., D.G.S., C.H., G.L., Y.Y., and S.S. gratefully acknowledge the financial support of the Semiconductor Research Corporation (SRC) and SEMATECH through the

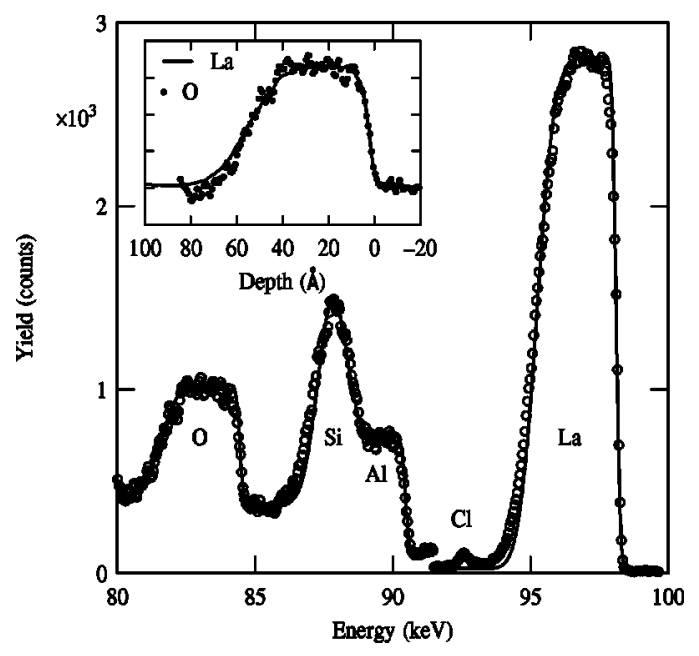

FIG. 5. MEIS energy spectrum of 40-Å-thick amorphous $\mathrm{LaAlO}_{3}$ on (001) $\mathrm{Si}$ along with the model calculated assuming an $\mathrm{SiO}_{2}$-free interface. Inset shows lanthanum and oxygen depth distributions.
SRC/SEMATECH FEP Center. L.F.E. gratefully acknowledges an AMD/SRC fellowship. The portion of this work conducted at PNNL was carried out in the Environmental Molecular Sciences Laboratory, a national scientific user facility sponsored by the Department of Energy's Office of Biological and Environmental Research.

${ }^{1}$ D. A. Muller, T. Sorsch, S. Moccio, F. H. Baumann, K. Evans-Lutterodt, and G. Timp, Nature (London) 399, 758 (1999).

${ }^{2}$ C. A. Billman, P. H. Tan, K. J. Hubbard, and D. G. Schlom, in Ultrathin $\mathrm{SiO}_{2}$ and High-K Materials for ULSI Gate Dielectrics, edited by H. R. Huff, C. A. Richter, M. L. Green, G. Lucovsky, and T. Hattori (Materials Research Society, Warrendale, PA, 1999), Vol. 567, pp. 409-414.

${ }^{3}$ A. I. Kingon, J.-P. Maria, and S. K. Streiffer, Nature (London) 406, 1032 (2000).

${ }^{4}$ D. G. Schlom and J. H. Haeni, MRS Bull. 27, 198 (2002).

${ }^{5}$ B.-E. Park and H. Ishiwara, Appl. Phys. Lett. 79, 806 (2001).

${ }^{6}$ B.-E. Park and H. Ishiwara, Appl. Phys. Lett. 82, 1197 (2003).

${ }^{7}$ X.-B. Lu, Z.-G. Liu, Y.-P. Wang, Y. Yang, X.-P. Wang, H.-W. Zhou, and B.-Y. Nguyen, J. Appl. Phys. 94, 1229 (2003).

${ }^{8}$ L. F. Edge, D. G. Schlom, S. A. Chambers, E. Cicerrella, J. L. Freeouf, B. Holländer, and J. Schubert, Appl. Phys. Lett. 84, 726 (2004).

${ }^{9}$ S. Stemmer and D. G. Schlom, in Nano and Giga Challenges in Microelectronics, edited by J. Greer, A. Korkin, and J. Labanowski (Elsevier, Amsterdam, 2003), pp. 129-150.

${ }^{10}$ International Technology Roadmap for Semiconductors: 2003 (Semiconductor Industry Association, San Jose, CA, 2003).

${ }^{11}$ S. Guha, E. Cartier, M. A. Gribelyuk, N. A. Bojarczuk, and M. C. Copel, Appl. Phys. Lett. 77, 2710 (2000).

${ }^{12}$ G. D. Wilk and R. M. Wallace, Appl. Phys. Lett. 76, 112 (2000).

${ }^{13}$ J. Kwo, M. Hong, A. R. Kortan, K. L. Queeney, Y. J. Chabal, R. L. Opila, D. A. Muller, S. N. G. Chu, B. J. Sapjeta, T. S. Lay, J. P. Mannaerts, T. Boone, H. W. Krautter, J. J. Krajewski, A. M. Seregent, and J. M. Rosamilia, J. Appl. Phys. 89, 3920 (2001).

${ }^{14}$ S. Stemmer, D. O. Klenov, Z. Chen, D. Niu, R. W. Ashcraft, and G. N. Parsons, Appl. Phys. Lett. 81, 712 (2002).

${ }^{15}$ W. Tsai, R. J. Carter, H. Nohira, M. Caymax, T. Conard, V. Cosnier, S. DeGendt, M. Heyns, J. Petry, O. Richard, W. Vandervorst, E. Young, C. Zhao, J. Maes, M. Tuominen, W. H. Schulte, E. Garfunkel, and T. Gustafsson, Microelectron. Eng. 65, 259 (2003).

${ }^{16}$ J. Kwo, M. Hong, B. Busch, D. A. Muller, Y. J. Chabal, A. R. Kortan, J. P. Mannaerts, B. Yang, P. Ye, H. Gossmann, A. M. Sergent, K. K. Ng, J. Bude, W. H. Schulte, E. Garfunkel, and T. Gustafsson, J. Cryst. Growth 251, 645 (2003)

${ }^{17}$ M. Ritala, K. Kukli, A. Rahtu, P. I. Räisänen, M. Leskelä, T. Sajavaara, and J. Keinonen, Science 288, 319 (2000).

${ }^{18}$ E. P. Gusev, M. Copel, E. Cartier, I. J. R. Baumvol, C. Krug, and M. A. Gribelyuk, Appl. Phys. Lett. 76, 176 (2000).

${ }^{19}$ S. Guha, E. Cartier, N. A. Bojarczuk, J. Bruley, L. Gignac, and J. Karasinski, J. Appl. Phys. 90, 512 (2001).

${ }^{20}$ S. J. Wang, C. K. Ong, S. Y. Xu, P. Chen, W. C. Tjiu, J. W. Chai, A. C. H. Huan, W. J. Yoo, J. S. Lim, W. Feng, and W. K. Choi, Appl. Phys. Lett. 78, 1604 (2001).

${ }^{21}$ S. J. Wang and C. K. Ong, Appl. Phys. Lett. 80, 2541 (2002).

${ }^{22}$ H. Li, X. Hu, Y. Wei, Z. Yu, X. Zhang, R. Droopad, A. A. Demkov, J. Edwards, K. Moore, W. Ooms, J. Kulik, and P. Fejes, J. Appl. Phys. 93, 4521 (2003).

${ }^{23}$ J. Y. Dai, P. F. Lee, K. H. Wong, H. L. W. Chen, and C. L. Choy, J. Appl. Phys. 94, 912 (2003).

${ }^{24}$ L. Yan, H. B. Lu, G. T. Tan, F. Chen, Y. L. Zhou, G. Z. Yang, W. Liu, and Z. H. Chen, Appl. Phys. A: Mater. Sci. Process. 77, 721 (2003).

${ }^{25}$ L. F. Edge and D. G. Schlom (unpublished).

${ }^{26}$ The nominal film thicknesses given were calculated from the fluxes of the molecular beams (measured by a quartz crystal microbalance) assuming the amorphous $\mathrm{LaAlO}_{3}$ films had the density of crystalline $\mathrm{LaAlO}_{3}$. The areal density $\left(\right.$ atoms $/ \mathrm{cm}^{2}$ ) of lanthanum and aluminum in the films was confirmed by RBS. The thicknesses of the amorphous $\mathrm{LaAlO}_{3}$ films were also measured by x-ray reflectivity and show 1.5-2 times the thicknesses determined assuming the density of crystalline $\mathrm{LaAlO}_{3}$. These differences will be discussed elsewhere

${ }^{27}$ J. Lettieri, J. H. Haeni, and D. G. Schlom, J. Vac. Sci. Technol. A 20, 1332 (2002).

${ }^{28}$ B. B. Stefanov, A. G. Gurevich, M. K. Weldon, K. Raghavachari, and Y. J. Chabal, Phys. Rev. Lett. 81, 3908 (1998). 